\title{
Successful use of supraglottic airway in a professional singer undergoing laparoscopic living donor right hepatectomy - A case report -
}

Received February 21, 2018

Revised April 24, 2018

Accepted April 27, 2018

\section{Corresponding author}

Gaab-Soo Kim, M.D., Ph.D.

Department of Anesthesiology and

Pain Medicine, Samsung Medical

Center, Sungkyunkwan University

School of Medicine, 81 Irwon-ro,

Gangnam-gu, Seoul 06351, Korea

Tel: 82-2-3410-0360

Fax: 82-2-3410-0361

E-mail: gskim@skku.edu

ORCID

http://orcid.org/0000-0002-9383-2652

\section{Jeayoun Kim, Gaab-Soo Kim, Duk Kyung Kim, and Hyunsu Shin}

Department of Anesthesiology and Pain Medicine, Samsung Medical Center, Sungkyunkwan University School of Medicine, Seoul, Korea
A 25-year old female singer was scheduled to undergo a right hepatectomy for her father's liver transplantation. Her two main requests were rapid recovery and prevention of cosmetic complications, and the avoidance of postoperative laryngeal damage. Thus, we decided to use a laparoscopic surgical approach and the second-generation supraglottic airway (Protector ${ }^{\mathrm{TM}}$ supraglottic airway). After anesthetic induction, the supraglottic airway was placed at the first attempt, and its performance was tested using the oropharyngeal leak pressure and maximal minute volume ventilation tests. Throughout the surgery, the cuff pressure of the supraglottic airway was maintained in the green zone. The Protector ${ }^{\mathrm{TM}}$ supraglottic airway was successfully used during 300 minutes of anesthesia, and it only caused mild postoperative sore throat without hoarseness or aspiration. Anesthesiologists should consider using the supraglottic airway proactively in laparoscopic living donor right hepatectomies when professional voice users undergo surgery.

Keywords: Hepatectomy; Laparoscopy; Laryngeal injury; Laryngeal masks; Living donor.
Recently, purely laparoscopic donor hepatectomy has been increasingly performed for its cosmetic and rapid recovery advantages. Because it requires a longer operative time and capnoperitoneum, it is preferred to use endotracheal intubation. This report presents our experience with using a supraglottic airway in a young female singer undergoing a laparoscopic donor right hepatectomy. Our case shows how the supraglottic airway can be a safe and effective alternative to endotracheal intubation during laparoscopic donor hepatectomy.

\section{CASE REPORT}

A 25-year-old female singer (weight $43.3 \mathrm{~kg}$, height 150.4 $\mathrm{cm}$ ) was scheduled to undergo a right hepatectomy for her father's liver transplantation. She elected the laparoscopic approach for rapid recovery and prevention of cosmetic complications. After we had a direct conversation with the patient, the use of the supraglottic airway was decided upon to prevent potential laryngeal damage after endotracheal intubation. After starting fasting at midnight the night before, the patient was transferred to the operating room. After instituting monitoring by standard devices, including pulse oximetry, 3-lead electrocardiography and non-invasive arterial blood pressure monitoring, $400 \mu \mathrm{g}$ of morphine sulfate (Hana Pharmaceutical Co., Ltd., Korea) was injected intrathecally for postoperative pain control. Anesthesia was induced with $300 \mathrm{mg}$ of thiopental sodium, and maintained with continu-

This is an Open Access article distributed under the terms of the Creative Commons Attribution Non-Commercial License (http://creativecommons.org/licenses/by-nc/4.0) which permits unrestricted non-commercial use, distribution, and reproduction in any medium, provided the original work is properly cited. 
ous infusion of vecuronium, remifentanil, and isoflurane in combination with $67 \%$ air in oxygen.

A second-generation supraglottic airway (Protector ${ }^{\mathrm{TM}}$ airway with cuff pilot technology, size 3, Teleflex Medical Europe, Ltd., Ireland) was inserted without difficulty. In order to confirm correct positioning of the tip of the supraglottic airway and to prevent possible gastric distention, a 14-French Levin tube was inserted through one of the airway's two gastric ports. The patient was positioned in the lithotomy position with the head slightly elevated. Then carbon dioxide gas was insufflated into the abdomen, and the intraabdominal pressure was maintained at approximately $10-12 \mathrm{mmHg}$.

The patient was mechanically ventilated with $6 \mathrm{ml} / \mathrm{kg}$ tidal volume [1] at a respiratory rate of $10-14 / \mathrm{min}$ to maintain normocapnia, at an I:E ratio of $1: 2$, with a fresh gas flow rate of $2 \mathrm{~L} / \mathrm{min}$. A positive end-expiratory pressure of $6 \mathrm{cmH}_{2} \mathrm{O}$ was maintained to prevent atelectasis.

After carbon dioxide insufflation, we performed oropharyngeal leak pressure and maximal minute volume ventilation tests. With the patient's head placed in the neutral position, the oropharyngeal leak pressure was determined by closing the expiratory valve of the circle system at a fixed gas flow of $4 \mathrm{~L} / \mathrm{min}$. The oropharyngeal leak pressure was deemed to be the pressure in the circuit when an audible noise was heard over the mouth and when there was equilibrium of airway pressure in the breathing circuit [2]. As a result, there was no audible leak until the peak airway pressure was $32 \mathrm{cmH}_{2} \mathrm{O}$.

A maximal minute volume ventilation test was performed by measuring 4 maximum breaths in 15 seconds using the reservoir bag [3], showing a result of $12.2 \mathrm{~L}$. Throughout the surgery, the cuff pressure of the supraglottic airway was maintained in the green zone to prevent pharyngo-laryngeal complications [4]. The cuff pressure measured by an airway pressure manometer (control inflator, VBM Medizintechnik $\mathrm{GmbH}$, Germany) was $30 \mathrm{cmH}_{2} \mathrm{O}$. Ventilatory failure did not occur.

Eleven $\mathrm{ml}$ of bile-colored gastric content was naturally drained. At the end of the operation, additional suction was applied to the Levin tube to prevent aspiration of gastric contents. The suctioned fluid was also bile-colored and $27 \mathrm{ml}$ in volume. Gastric distension was not observed in the surgical field intraoperatively. Postoperatively, the supraglottic airway was removed with the cuff deflated when the patient was able to open her eyes to command. No blood was observed on the surface of the supraglottic airway. A small amount of bile-colored fluid was identified on the surface of the supraglottic airway, which was insignificant and mostly located in the gastric port. The total durations of pneumoperitoneum and supraglottic airway insertion were 188 and 300 minutes, respectively. The crystalloid solution used for infusion was 1,950 ml of Plasma solution A (CJ HealthCare Corp., Korea), and urine output was $171 \mathrm{ml}$. Intraoperative blood loss was approximately $340 \mathrm{ml}$.

The patient was transferred to the post-anesthesia care unit where she experienced only mild sore throat and postoperative pain. Her sore throat was eventually relieved by postoperative day 1 . She did not complain of hoarseness during her hospital stay and was discharged on postoperative day 9 .

\section{DISCUSSION}

Minimization of perioperative complications is crucial during donor hepatectomy, as the donors are relatively young and healthy people. Recently, purely laparoscopic donor right hepatectomy has been increasingly performed for its cosmetic and rapid recovery advantages. However, it is known to be technically difficult and requires a longer operative time [5]. Our patient was scheduled to undergo purely laparoscopic donor right hepatectomy, which takes approximately 6 hours at our institution.

Because the patient was a professional singer, the most feared complication was vocal cord or laryngeal injury, which made it undesirable to intubate her using an endotracheal tube. Endotracheal intubation is associated with a significant rate of laryngeal damage and subsequent vocal pathology. According to the American Society of Anesthesiologists' Closed Claims database [6], approximately $6 \%$ of claims were airway injury during general anesthesia with endotracheal intubation. The most common site of airway injury was the larynx, representing $33 \%$ of all airway injury claims. The most frequent types of laryngeal injury were vocal cord paralysis and hematoma or granuloma of the vocal cords. Risk factors include female sex, elective surgery, and outpatient procedures. Kikura et al. [7] reported that the risk of vocal cord paralysis increases three-fold in patients aged 50 years or older, 15-fold in patients intubated for 6 hours or more, and two-fold in patients with a history of diabetes mellitus or hypertension. Our patient had several risk factors for vocal cord 
injury including female sex and long duration of surgery.

In contrast to endotracheal intubation, placement of the supraglottic airway involves no laryngeal passage, and it is, therefore, associated with a significantly reduced incidence of postoperative laryngeal damage. One systemic review of randomized, controlled trials revealed that the use of the supraglottic airway resulted in a statistically and clinically significant lower incidence of laryngospasm during emergence, postoperative hoarseness, sore throat, and coughing than the use of endotracheal tubes [8]. Therefore, we elected to use the supraglottic airway in this patient to reduce the probability of laryngeal complications. However, the effectiveness of the supraglottic airway is significantly limited in laparoscopic surgeries, and further limited during prolonged anesthesia [9]. In addition, there is a theoretical risk of aspiration and leakage of air during positive pressure ventilation because the esophagus and airway are directly connected when the supraglottic airway is inserted [10]. Laparoscopic donor right hepatectomy requires a long operating time, as it lasts 6 hours on average at out institution; therefore, it is not a conventional indication for supraglottic airway use. However, recently developed second-generation supraglottic airways enable the application of higher respiratory pressure, drainage of regurgitant material, and insertion of a gastric tube via the integrated gastric access. These developments have expanded its indications for instances such as prolonged periods of use and minor laparoscopic procedures [3]. In addition, according to Bernardini and Natalini [11], pulmonary aspiration during supraglottic airway use is uncommon and comparable to that for outpatient anesthesia with a face mask and endotracheal tube. In a review of 11,910 cases, the supraglottic airway appeared to be safe during gynecologic laparoscopic surgery and anesthesia lasting over 2 hours [12]. Other case reports have described the unexpectedly safe usage of second-generation supraglottic airways for 7 hours and 20 minutes in a parotid gland tumor excision and 9 hours in a multiple traumatic patient before surgical airway achievement $[13,14]$. Therefore, despite the prolonged anesthesia and laparoscopic approach involved, our decision to use this approach achieved successful anesthetic management without any postoperative laryngeal complications [11-14].

In order to minimize the possible risk of aspiration, the patient fasted from midnight on the day before surgery, and two performance tests (oropharyngeal leak pressure and maximal minute volume ventilation tests) were performed to check the position of the placed supraglottic airway. In addition, a Levin tube was inserted through the gastric port of the supraglottic airway to confirm proper positioning and to drain the gastric contents during surgery. The laparoscopic monitor was used to identify possible gastric distention, which could cause gastric regurgitation during surgery. Finally, the Levin tube was suctioned just before removal of the supraglottic airway to prevent gastric contents passing to the airway. Despite these efforts, a small amount of the bile-colored regurgitant was observed on the outer surface of the supraglottic airway. However, it was mainly observed in the gastric port of the supraglottic airway, and its amount was not significant. It seemed to have flowed from the end of the Levin tube as it was removed from the stomach just before removal of the supraglottic airway. As a result, pulmonary complications related to aspiration did not occur.

According to the cuff pressure monitoring system of the Protector $^{\mathrm{TM}}$ supraglottic airway (termed cuff pilot technology), cuff pressure in the green zone is $40-60 \mathrm{cmH}_{2} \mathrm{O}$. In this case, the patient experienced a transient episode of sore throat, which might have been caused by the prolonged pressure applied to the oropharynx in the area where the supraglottic airway was supported [4]. However, the sore throat was mild in severity and resolved with no residual discomfort a day later.

In conclusion, this case shows the feasibility of the supraglottic airway as an alternative to endotracheal intubation during prolonged laparoscopic living donor right hepatectomy. Its use should be considered in laparoscopic living donor hepatectomies when professional voice users undergo surgery.

\section{REFERENCES}

1. Futier E, Constantin JM, Paugam-Burtz C, Pascal J, Eurin M, Neuschwander A, et al. A trial of intraoperative low-tidal-volume ventilation in abdominal surgery. N Engl J Med 2013; 369: 42837.

2. Keller C, Brimacombe JR, Keller K, Morris R. Comparison of four methods for assessing airway sealing pressure with the laryngeal mask airway in adult patients. Br J Anaesth 1999; 82: 286-7.

3. Timmermann A, Bergner UA, Russo SG. Laryngeal mask airway indications: new frontiers for second-generation supraglottic airways. Curr Opin Anaesthesiol 2015; 28: 717-26. 
4. Seet E, Yousaf F, Gupta S, Subramanyam R, Wong DT, Chung F. Use of manometry for laryngeal mask airway reduces postoperative pharyngolaryngeal adverse events: a prospective, randomized trial. Anesthesiology 2010; 112: 652-7.

5. Kim KH, Kang SH, Jung DH, Yoon YI, Kim WJ, Shin MH, et al. Initial outcomes of pure laparoscopic living donor right hepatectomy in an experienced adult living donor liver transplant center. Transplantation 2017; 101: 1106-10.

6. Domino KB, Posner KL, Caplan RA, Cheney FW. Airway injury during anesthesia: a closed claims analysis. Anesthesiology 1999; 91: 1703-11.

7. Kikura M, Suzuki K, Itagaki T, Takada T, Sato S. Age and comorbidity as risk factors for vocal cord paralysis associated with tracheal intubation. Br J Anaesth 2007; 98: 524-30.

8. Yu SH, Beirne OR. Laryngeal mask airways have a lower risk of airway complications compared with endotracheal intubation: a systematic review. J Oral Maxillofac Surg 2010; 68: 2359-76.
9. Asai T, Morris S. The laryngeal mask airway: its features, effects and role. Can J Anaesth 1994; 41: 930-60.

10. Payne J. The use of the fibreoptic laryngoscope to confirm the position of the laryngeal mask. Anaesthesia 1989; 44: 865.

11. Bernardini A, Natalini G. Risk of pulmonary aspiration with laryngeal mask airway and tracheal tube: analysis on 65712 procedures with positive pressure ventilation. Anaesthesia 2009; 64: 1289-94.

12. Verghese C, Brimacombe JR. Survey of laryngeal mask airway usage in 11,910 patients: safety and efficacy for conventional and nonconventional usage. Anesth Analg 1996; 82: 129-33.

13. Braude D, Southard A, Bajema T, Sims E, Martinez J. Rapid sequence airway using the LMA-Supreme as a primary airway for 9 $\mathrm{h}$ in a multi-system trauma patient. Resuscitation 2010; 81: 1217.

14. Kammah M, Añez C. Prolonged use of the LMA Supreme ${ }^{\mathrm{TM}}$. Can J Anaesth 2013; 60: 411-2. 